\title{
Salmonella Typhimurium lacking the Znuabc transporter is attenuated and immunogenic in pigs
}

\author{
M. Pesciaroli a , M. Gradassi ${ }^{\mathrm{b}}$, N. Martinelli $^{\mathrm{b}}$, J. Ruggeri $^{\mathrm{b}}$, C. Pistoia ${ }^{\mathrm{a}}$, M. Raffatellu ${ }^{\mathrm{c}}$, C.F. Magistrali ${ }^{\mathrm{d}}$, \\ A. Battistoni ${ }^{\text {e }}$, P. Pasquali ${ }^{\mathrm{a}, *}$, G.L. Alborali ${ }^{\mathrm{b}}$ \\ a Department of Veterinary Public Health and Food Safety, Istituto Superiore di Sanità, 00161 Rome, Italy \\ b Istituto Zooprofilattico Sperimentale della Lombardia e dell'Emilia Romagna, 25124 Brescia, Italy \\ ${ }^{c}$ Department of Microbiology and Molecular Genetics, University of California, Irvine, CA 92697, USA \\ d Istituto Zooprofilattico Sperimentale dell'Umbria e delle Marche, 06126 Perugia, Italy \\ e Department of Biology, Università di Roma Tor Vergata, 00133 Rome, Italy
}

\section{A R T I C L E I N F O}

\section{Article history:}

Received 22 January 2013

Received in revised form 8 April 2013

Accepted 11 April 2013

Available online 24 April 2013

\section{Keywords:}

Vaccine

Salmonella

Pig

Safety

Immunogenicity

\begin{abstract}
A B S T R A C T
Meat contamination by Salmonella spp. is emerging as a major cause of human enteric infections in industrialized countries. The attempts to reduce human cases of salmonellosis encompass pre- and post-harvest interventions. In this context, vaccination of pigs may represent an effective instrument in eliminating/reducing Salmonella burden through the food chain. We have previously demonstrated that Salmonella Typhimurium lacking the ZnuABC transporter (S. Typhimurium $\triangle z n u A B C$ ) is a promising candidate live vaccine in different mouse models of Salmonella Typhimurium infection. In this study, we confirmed in pigs the attenuation of S. Typhimurium $\triangle z n u A B C$. Moreover, we evaluated the safety and immunogenicity of $S$. Typhimurium $\triangle z n u A B C$ administered to pigs by the oral route. We monitored clinical conditions of animals and we conducted a microbiological culture and a quantification of the humoral and cellular immune response, respectively, on fecal and blood samples of pigs. After vaccination with attenuated $S$. Typhimurium $\triangle z n u A B C$, pigs showed a modest degree of hyperthermia. In addition, fecal shedding of $S$. Typhimurium $\triangle Z n u A B C$ could not be detected 28 days after the inoculum. Furthermore, vaccination with $S$. Typhimurium $\triangle \mathrm{znu} A B C$ elicited a distinct production of anti-Salmonella antibodies and IFN- $\gamma$. Taken together, these results suggest that $S$. Typhimurium $\triangle z n u A B C$ is attenuated and immunogenic in pigs. Although the vaccine dosages do not guarantee complete safety there is ample margin to set up better conditions of use, suggesting that $S$. Typhimurium $\triangle z n u A B C$ could be a promising attenuated strain to be used as live mucosal vaccine for oral delivery.
\end{abstract}

(c) 2013 Elsevier Ltd. All rights reserved.

\section{Introduction}

Nontyphoidal salmonellosis infections pose a significant threat to human health and are responsible for high economic losses worldwide [1]. Moreover, many Salmonella strains are resistant to a number of antimicrobial agents, narrowing the therapeutic alternatives in cases of severe human infection [2,3]. Pork is the main source of Salmonella infection for humans, accounting for $26.9 \%$ of the human cases officially reported in the EU [4]. The application of strict hygiene practices and rational husbandry management have been effective in Scandinavian countries, where the prevalence of Salmonella in animals and carcasses at slaughter is nearly zero. However, this approach is hardly feasible in countries where high prevalence of infection is observed. In these settings,

\footnotetext{
* Corresponding author. Tel.: +39 06 49902326; fax: +39 0649387077.

E-mail address: paolo.pasquali@iss.it (P. Pasquali).
}

vaccination is considered as a major tool to minimize Salmonella contamination at the early stages of meat production. Although live vaccines provide better protection against Salmonella infections compared to inactivated ones [5-8], only a live attenuated vaccine for Salmonella Typhimurium is commercially available in Europe at the moment [5]. Nevertheless, several strains showed promising results vaccine in experimental settings [6-8]. Recently, we found that a Salmonella enterica serovar Typhimurium mutant strain, deleted of the whole $z n u A B C$ operon (S. Typhimurium $\triangle z n u A B C$ ) encoding for a high affinity zinc importer necessary for metal recruitment within the infected host, is significantly attenuated in mice [9-11] and able to protect mice against both systemic and enteric salmonellosis [12,13]. Based on these results, compared the virulence of $S$. Typhimurium $\triangle z n u A B C$ with those of wild type Salmonella Typhimurium ATCC 14028 in pigs (Exp. 1). In a second experiment (Exp. 2), we established the safety and immunogenicity of $S$. Typhimurium $\triangle z n u A B C$ administered to pigs as live vaccine. The results reported here demonstrate that $S$. Typhimurium 
$\triangle z n u A B C$ is attenuated in pigs. Moreover, administered by the oral route, $S$. Typhimurium $\triangle \mathrm{znu} A B C$ elicits a short-lasting and immunogenic infection that does not affect the animal health status and production performances in nearly all animals.

\section{Materials and methods}

\subsection{Salmonella spp. cultures}

The virulent Salmonella Typhimurium ATCC 14028 and its isogenic $z n u A B C$ mutant strain produced according to the method previously reported [9], were used throughout the study. Strains were grown overnight at $37^{\circ} \mathrm{C}$ in Brain Heart Infusion (Oxoid Ltd., UK), harvested by centrifugation and then washed twice in ice-cold phosphate buffer solution (PBS) (Sigma-Aldrich, Italy). A bacterin from $S$. Typhimurium ATCC 14028 was obtained by inactivating the bacteria with formalin and absorbing them on aluminum hydroxide.

\subsection{Animals}

Twenty-eight commercial hybrid pigs aging $\sim 80$ days were used in the comparative virulence experiment (Exp. 1). Animals were split in two groups of 10 (Groups A and B) and a group of 8 (Group C). Groups A and B were intragastrically administered with $20 \mathrm{ml}$ of sodium bicarbonate buffer containing $5 \times 10^{9} \mathrm{CFU}$ of $S$. Typhimurium $\triangle z n u A B C$ (Group A) or $5 \times 10^{9} \mathrm{CFU}$ of $S$. Typhimurium ATCC 14028 (Group B). Group C received only sterile sodium bicarbonate buffer and served as control group. Collection of fecal samples of each pig ( $0,1,3$ and 7 days) and registration of rectal temperature $(0,1,2$ and 7 days) were performed. For the safety and immunogenicity experiment (Exp. 2), hybrid pigs born by cesarean section and aging 80-100 days were used. Animals were split into 4 groups of 6 (Groups A, B and C) and 8 (Group D) animals/group. Groups A and B were intragastrically vaccinated, respectively, with a suspension of $5 \times 10^{8}$ (Group A) and $5 \times 10^{7}$ (Group B) CFU of $S$. Typhimurium $\triangle z n u A B C$ in $20 \mathrm{ml}$ of sodium bicarbonate buffer. Group $C$ was intramuscularly (upper part of the neck, $16 \mathrm{G}$ needle, $40 \mathrm{~mm}$ length) vaccinated with $2 \times 10^{9} \mathrm{CFU}$ of inactivated S. Typhimurium ATCC 14028 . Group C received an equal booster dose 14 days later. Group D was administered $20 \mathrm{ml}$ of sterile sodium bicarbonate buffer and was left as naïve controls. Individual blood and fecal samples were collected before and after immunization (on day $0,1,2,7,8,9,14,21,28$ and 35 days). Body temperature was registered on the same dates. On day 0 and at 5 weeks, each animal was weighed to evaluate the growth rate. All the pigs used throughout the study (Exp. 1 and Exp. 2) were proved to be Salmonella-free by culture of feces of each animal, and individual sera were checked to exclude Salmonella antibodies. Each group was maintained in separate isolation units under natural day-night rhythm with access to feed (FAMAVIT, Italy) and water ad libitum.

All the experiments were authorized by national authority and were conducted according to national regulation (D.L.116/92)

\subsection{Fecal shedding of S. Typhimurium}

Fecal samples of each pig were collected to assess the elimination of bacteria. The microbiological analysis was conducted according to the ISO 6579:2002/Amendment 1:2007 protocol. This is a semi-quantitative approach that allowed determining the concentration of Salmonella in a sample within a tenfold band (detection limit $1 \mathrm{CFU} / \mathrm{g}$ feces). Suspect Salmonella colonies were subjected to biochemical identification by BBL Enterotube II (BD Franklin Lakes, USA) and serological identification using Salmonella group-specific antisera (Remel, Lenexa, USA).

\subsection{Persistence of S. Typhimurium $\triangle$ znuABC in the environment and pig feces}

Environmental swabs collected from the pen floor of each group were qualitatively cultured following the protocol indicated in section 2.3 to assess the persistence of Salmonella in the environment. In another set of experiments, we compared the viability of $S$. Typhimurium $\triangle z n u A B C$ and $S$. Typhimurium ATCC 14028 in feces. Approximately $2 \mathrm{~kg}$ of fresh pig feces were collected from a group of sows serologically negative for Salmonella. Prior to use, the feces were confirmed negative for the presence of Salmonella by culture. A quantity of $27 \mathrm{~g}$ of feces was placed into each of 22 sterile $50 \mathrm{ml}$ plastic tubes for inoculation. Three $\mathrm{ml}$ of sterile saline containing $S$. Typhimurium $\triangle z n u A B C$ or $S$. Typhimurium ATCC 14028 were added to 20 tubes ( 10 tubes for each strain), to yield a final concentration of $5 \times 10^{6} \mathrm{CFU} / \mathrm{g}$ of feces. Finally, $3 \mathrm{ml}$ of sterile saline were added to 2 tubes, to be used as controls. Tubes were incubated at $20^{\circ} \mathrm{C}$ for three weeks. Sampling for culture was performed at $0,2,7,14$ and 21 days post-inoculation. After gently mixing, an aliquot of $1 \mathrm{~g}$ of feces was taken from each tube for enumeration of Salmonella microorganisms using a miniaturized MPN (Most Probable Number) method based on ISO 6579:2002 [14].

Typical colonies were confirmed serologically as Salmonella by polyvalent antiserum (Salmonella Test Serum; Siemens Healthcare Diagnostics, Italy) and API rapid 20 E (Api Rapid 20E; Biomerieux, Italy). MPN was calculated according to the ISO 7218:2007.

\subsection{Serological examination}

The serological exams were performed using a commercial indirect ELISA test capable of detecting antibodies against lipopolysaccharide antigens of Salmonella serogroups B, C1 and D (Herd-Check Swine Salmonella Antibody Test Kit, Idexx Laboratories Inc., Switzerland). The test was carried out according to the producer's instructions and read at an optical density of $450 \mathrm{~nm}$. Results were expressed as sample to positive ratio $\{\mathrm{S}: \mathrm{P}$ ratio $=(\mathrm{OD}$ of sample - OD of negative control)/(OD of positive control - OD of negative control)\}.

\subsection{Statistical analysis}

Exp. 1. Differences in body temperature were estimated using 2 ways analysis of variance with a Tuckey's multiple comparison test and considered significant when ${ }^{*} P$-value $\leq 0.05$. Fecal shedding of group A and group B were compared using non parametric Mann-Whitney test.

Exp. 2. Differences between the groups were analyzed by a Twoway ANOVA and Bonferroni corrections were applied.

Statistical analysis was performed using a Graphpad PRISM 6.0 software (GraphPad Software Inc., USA). A $P$-value $\leq 0.05$ was considered significant.

\section{Results}

\subsection{Pathogenicity of S. Typhimurium $\triangle z n u A B C$}

Our hypothesis was that the deletion of the whole $z n u A B C$ operon encoding for a zinc importer necessary for metal recruitment within the infected host was able to reduce virulence of $S$. Typhimurium in pigs. So, in Exp.1, we compared the values of body temperature and the duration of fecal shedding of group $A$ (S. Typhimurium $\triangle z n u A B C$ ) with those of Group B (S. Typhimurium ATCC 14028) and Group C (controls). Two days after the inoculation, group A and group B displayed statistically significant higher values of body temperature than controls. Nevertheless the increase body temperature was more evident in group B (Fig. 1A). S. Typhimurium 


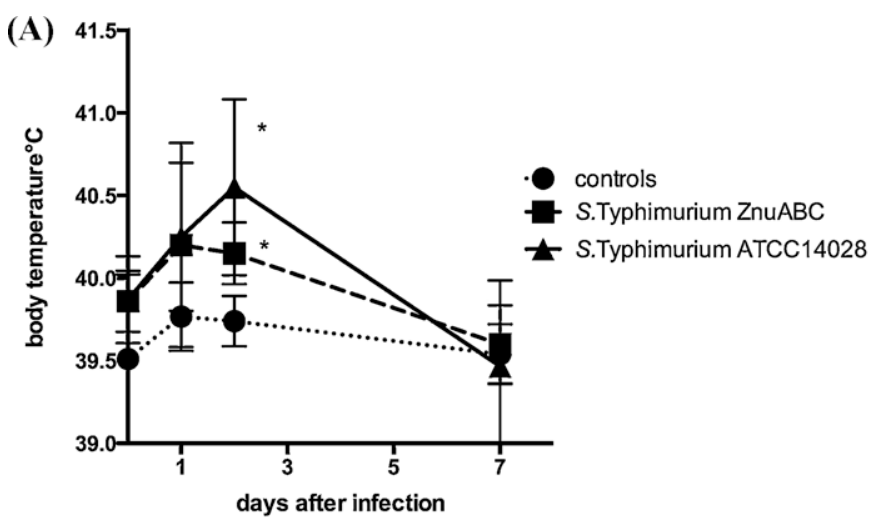

(B)

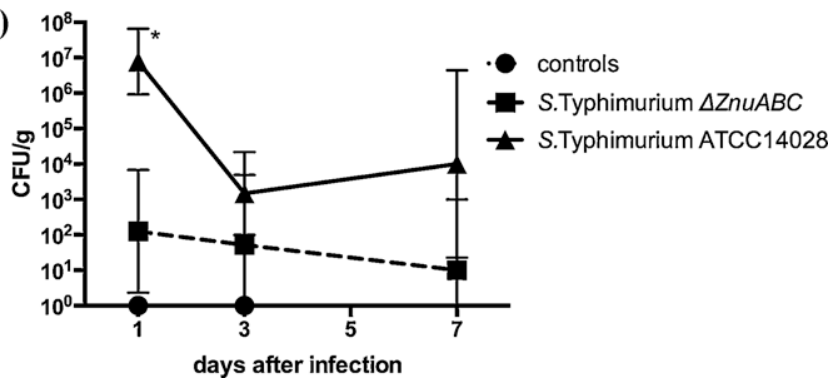

Fig. 1. $S$. Typhimurium $\triangle z n u A B C$ shows lower virulence in pigs compared to wild type $S$. Typhimurium ATCC 14028. (A) Mean values and SD bars of body temperature of groups $(A-C)$ at different time points. Results of group A or B were compared with results of group C. Differences were estimated using 2 ways analysis of variance with a Tuckey's multiple comparison test and considered significant when ${ }^{*} P$-value $\leq 0.05$. (B) Mean values and SD bars of CFU/g of $S$. Typhimurium $\triangle z n u A B C$ (group A) and $S$. Typhimurium ATCC14028 (group B) shed in feces. Results of group A were compared with results of group B. Differences were estimated using non parametric Mann Whitney test and were considered significant when ${ }^{*} P$-value $\leq 0.05$.

$\triangle z n u A B C$ was fecally shed in lower amount than $S$. Typhimurium ATCC 14028 (Fig. 1B). At 1 day after the inoculation this difference reached statistically significance. These results confirmed our hypothesis a $S$. Typhimurium deleted of whole znuABC operon has a reduced virulence in pigs.

\subsection{Effect of S. Typhimurium $\triangle$ znuABC oral administration on body temperature}

Then, in Exp. 2, we decided to explore how the oral vaccination with $S$. Typhimurium $\triangle Z n u A B C$ (our candidate vaccine strain mutated in $z n u A B C$ ) modifies the physiologic values of body temperature in pigs, considered to be between 38 and $40^{\circ} \mathrm{C}$ [15]. At 1 day after vaccination (DAV), three animals of group B and one animal of group A displayed a body temperature ranging from $40.3^{\circ} \mathrm{C}$ and $40.7^{\circ} \mathrm{C}$ (Fig. 2 and Fig. S1A). At 2 DAV, three animals of group $A$ and one animal of group $B$ had a body temperature varying from $40.1^{\circ} \mathrm{C}$ and $41^{\circ} \mathrm{C}$ (Fig. 2 and Fig. S1B). At the following time points (7, $14,21,28,35 \mathrm{DAV})$, the body temperature of both groups of pigs was comparable with controls. Group C, vaccinated with $2 \times 10^{9} \mathrm{CFU}$ of bacterin, and group $\mathrm{D}$ (controls) did not show any alteration of temperature at any time points considered (Fig. 2 and Fig. S1C and D). These data indicate that the oral administration of $S$. Typhimurium $\triangle z n u A B C$ at currently used inoculation doses increases body temperature of a limited number of pigs.

\subsection{Influence of vaccination on growth rate}

Next, we evaluated if vaccination with either $S$. Typhimurium $\triangle z n u A B C$ or inactivated $S$. Typhimurium ATCC 14028 interferes with the growth of animals. At $35 \mathrm{DAV}$, one animal of group

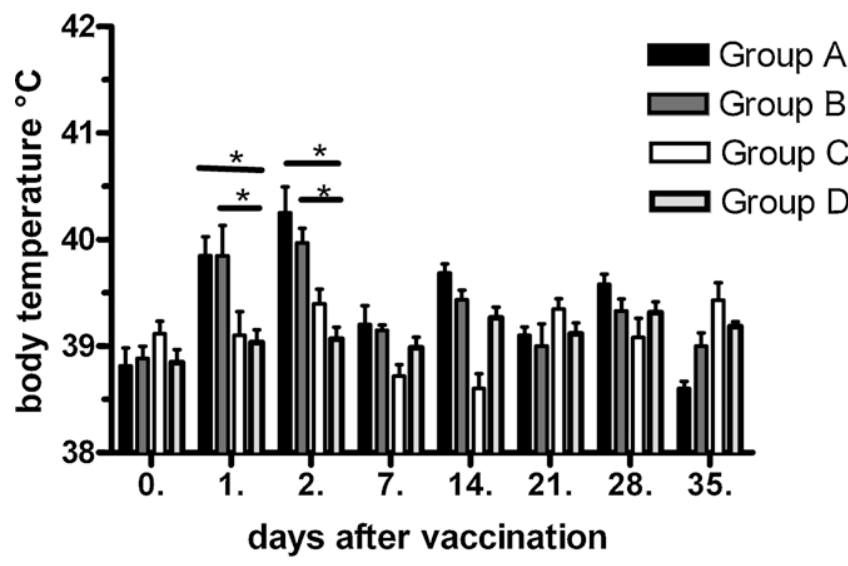

Fig. 2. Group A and group B display a rise of body temperature after vaccination with $S$. Typhimurium $\triangle z n u A B C$. Mean values and SD bars of body temperature of each group at different time points. Two-way ANOVA and Bonferroni corrections were applied. ${ }^{*} P$-value $\leq 0.05$.

B and one of group C did not gain weight, a decrease of body weight was registered in a pig of group A. Our observations suggest pigs of groups vaccinated either with $S$. Typhimurium $\triangle z n u A B C$ or inactivated $S$. Typhimurium ATCC 14028 showed an heterogeneous growth rate. However, no statistically significant difference was noted between the growth rate of vaccinated pigs and controls (Fig. 3 and Fig. S2).

\subsection{Fecal shedding of S. Typhimurium $\triangle z n u A B C$}

At 1 DAV, stools of group A contained approximately $10^{5} \mathrm{CFU} / \mathrm{g}$ of $S$. Typhimurium $\triangle z n u A B C$ and stools of group B contained less than $10^{4} \mathrm{CFU} / \mathrm{g}$ (Fig. 4). Twenty-four hours later, the concentration decreased by $\sim 2$ logs in feces of pigs from both groups. At 7 DAV, group A continued to excrete approximately $10^{2} \mathrm{CFU} / \mathrm{g}$. However, at 7 DAV, shedding of group B dropped to less than $10 \mathrm{CFU} / \mathrm{g}$, with 3 animals in this group that had not detectable $S$. Typhimurium $\triangle z n u A B C$ in feces. At 21 DAV, one animal of group B shed less than $10 \mathrm{CFU} / \mathrm{g}$ of $S$. Typhimurium $\triangle z n u A B C$. At $14 \mathrm{DAV}$, two pigs of group A shed less than $10 \mathrm{CFU} / \mathrm{g}$ and at $21 \mathrm{DAV}$, two other pigs excreted 10

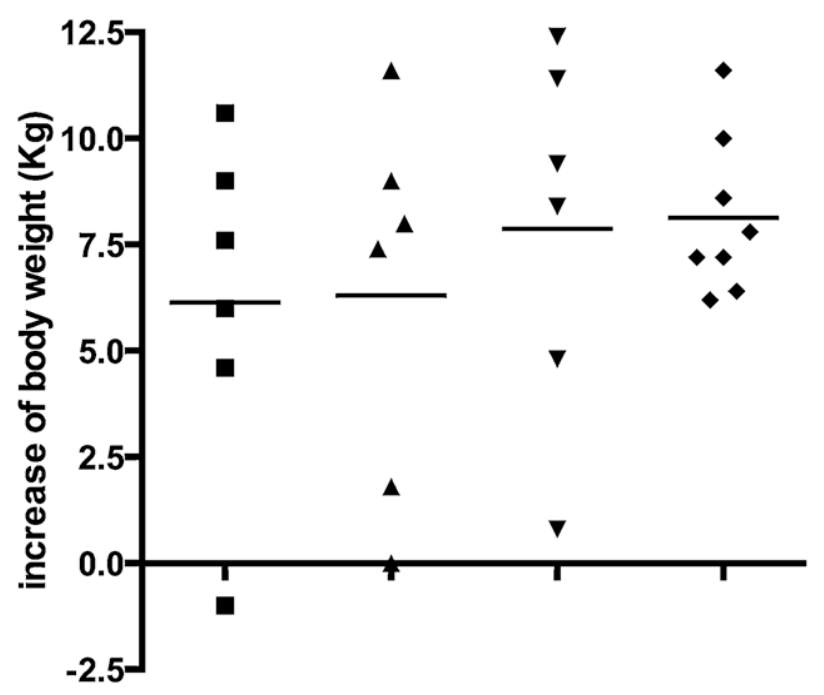

Group A Group B Group C Group D

Fig. 3. The weight gain of pigs of vaccinated groups is heterogeneous. Weight gain of animals of different groups. Each symbol represents one animal and bars represent mean value of the group. 


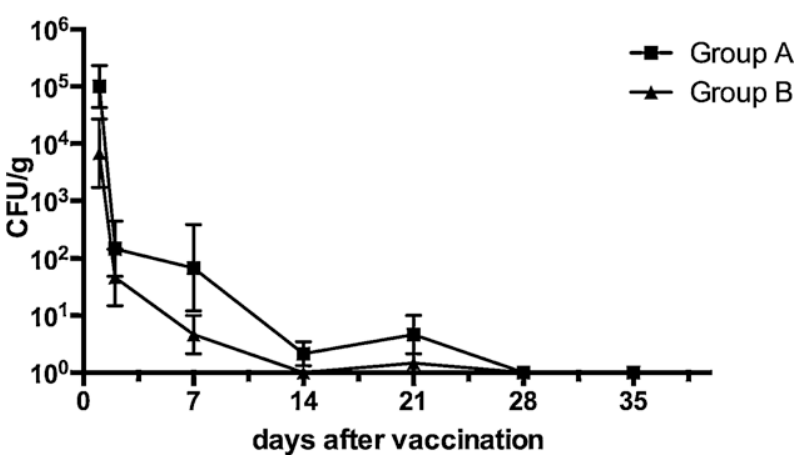

Fig. 4. Fecal shedding of $S$. Typhimurium $\triangle z n u A B C$. Fecal elimination of $S$. Typhimurium $\triangle z n u A B C$ by group $\mathrm{A}$ and group $\mathrm{B}$ after oral administration respectively with a dose of $5 \times 10^{8}$ and $5 \times 10^{7} \mathrm{CFU} / \mathrm{pig}$. Mean values with SD bars of CFU/g of each group at different time points.

and $10^{2} \mathrm{CFU} / \mathrm{g}$ of $S$. Typhimurium $\triangle z n u A B C$ each. The shedding of $S$. Typhimurium $\triangle z n u A B C$ by animals of group A was not detectable at $28 \mathrm{DAV}$. These data indicate that at $2 \mathrm{DAV}$, the fecal shedding of $S$. Typhimurium $\triangle z n u A B C$ decreases to approximately $10^{1}-10^{2} \mathrm{CFU} / \mathrm{g}$ and continues to comparable values till day 28 .

\subsection{Viability of S. Typhimurium $\triangle \mathrm{znuABC}$ in the environment and pig feces}

As indicator of the viability of $S$. Typhimurium $\triangle z n u A B C$, we assessed the environmental contamination of pig units after vaccination. As expected, $S$. Typhimurium $\triangle z n u A B C$ was not detected in pen of controls (group D) and animals of group C, which were vaccinated with the bacterin. Environmental swabs collected from pens of groups $A$ and $B$ revealed that $S$. Typhimurium $\triangle z n u A B C$ contaminated the environment till $7 \mathrm{DPI}$. To corroborate these findings we set out to determine the survival of $S$. Typhimurium $\triangle z n u A B C$ in comparison with $S$. Typhimurium ATCC 14028 in a fecal matrix. In this setting, the concentration of $S$. Typhimurium $\triangle z n u A B C$ dropped from $4 \times 10^{6} \mathrm{CFU} / \mathrm{g}$ (Standard Deviation $1.4 \times 10^{4}$ ) to 844 (Standard Deviation $8.9 \times 10^{2}$ ) in two days and was under the detection limit at 14 days after inoculation in the majority of the samples. In contrast, S. Typhimurium ATCC 14028 was still detectable at a concentration of $1.4 \times 10^{6}$ at 14 days after inoculum (Fig. 5). These data suggest that $S$. Typhimurium $\triangle z n u A B C$ does not persist long in conditions resembling the natural environment.

\subsection{Vaccination induces antibody specific immune response}

The antibody response of pigs after vaccination with $S$. Typhimurium $\triangle$ znuABC or inactivated S. Typhimurium ATCC 14028 was investigated. All vaccinated animals mounted a humoral immune response regardless of the type of vaccine administrated

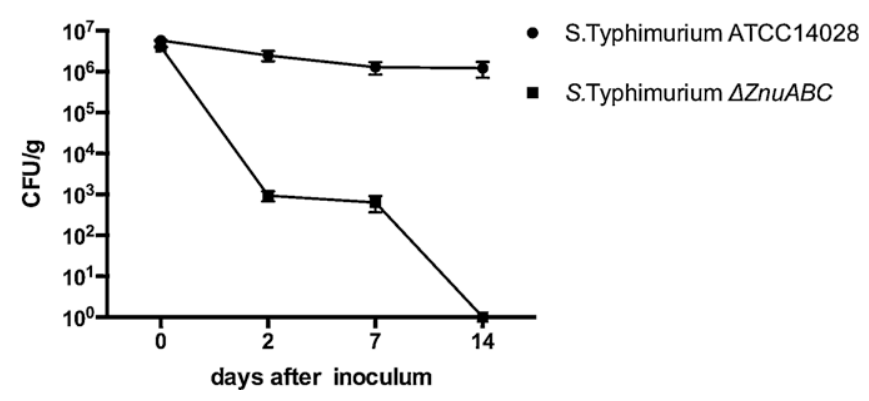

Fig. 5. S. Typhimurium $\triangle z n u A B C$ has a limited survival in fecal material. Survival of $S$. Typhimurium $\triangle z n u A B C$ and $S$. Typhimurium ATCC 14028 in artificial contaminated feces maintained at room temperature.

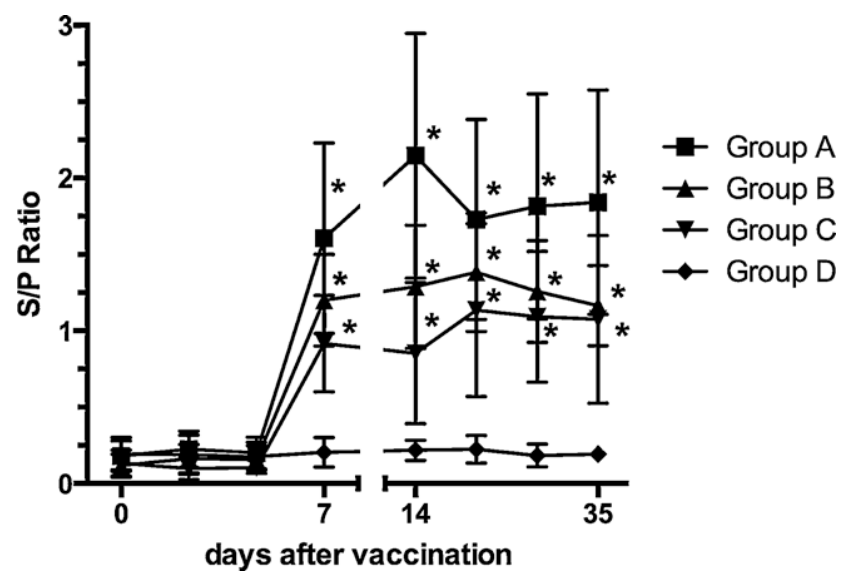

Fig. 6. $S$. Typhimurium $\triangle z n u A B C$ and inactivated S. Typhimurium ATCC14028 induce a similar pattern of humoral immune response. After vaccination, serum of pigs was collected at several time points; a commercially available ELISA test was used to detect antibodies against Salmonella. The X-axis (time) of figure is divided in two segments. Each segment has different intervals coinciding with time points. This solution was adopted to ameliorate the graphical illustration of data. Mean values with SD bars of antibodies titers of each group at different time points. Groups with asterisk are statistically different from group $D$ at the time point considered ${ }^{*} P$-value $\leq 0.05$.

(Fig. 6). Anti-Salmonella antibodies raised in group A, group B and group $C$ at $7 \mathrm{DAV}$ and remained high throughout the observational period. At 7, 14, 21, 28 and 35 DAV, antibody titers of groups A, B and $C$ were statistically higher than those of controls. These data demonstrate that administration of $S$. Typhimurium $\triangle z n u A B C$ or inactivated S. Typhimurium ATCC 14028 is effective in inducing a humoral immune response in pigs.

\section{Discussion}

The here reported results show that the mutant strain $S$. Typhimurium lacking the ZnuABC transporter inoculated in pigs by oral route shows characteristics of attenuation if compared with virulent $S$. Typhimurium ATCC 14028. Moreover, vaccination with $S$. Typhimurium $\triangle z n u A B C$ (our candidate vaccine strain mutated in $z n u A B C$ ) caused an increase of body temperature for two days but there was no difference in body temperature between the groups by $7 \mathrm{DAV})$. A heterogeneous growth rate within the vaccinated groups was noticed. However, mean of the weights of vaccinated groups were comparable with those of controls. S. Typhimurium $\triangle z n u A B C$ primed a specific humoral and cellular immune response (Supplementary Data). On the whole, these findings demonstrate that $S$. Typhimurium $\triangle z n u A B C$ has appreciable characteristics of safety and immunogenicity in pigs.

Vaccination is one of the methods suggested to decrease the burden of $S$. Typhimurium at swine farm level and prevent its dissemination through the pork production chain $[5,16,17]$.

To assess the safety of $S$. Typhimurium $\triangle z n u A B C$ in pigs we took into account several parameters such as body temperature, weight gain, serum concentration of TNF- $\alpha$ and fecal shedding of the vaccine strain.

Some vaccinated animals showed a rise in body temperature at 1 and 2 DAV, however it ended within one week. The administration of $S$. Typhimurium $\triangle z n u A B C$ caused stunted growth of some pigs regardless of vaccine dose. Nevertheless, body weight means of the experimental groups were comparable. However conclusive assessment requires further studies enrolling larger groups of animals with an homogeneous initial size and age, since a previous study reported that the administration of Salmonella live vaccines decreased the growth rate of vaccinated pigs [18]. 
The serum concentration of TNF- $\alpha$ in pigs inoculated with $S$. Typhimurium $\triangle z n u A B C$ showed a significant increase compared to controls only when the highest vaccinal dose is administered (Fig. S4) These data are consistent with previous observations that report reduced variations of serum concentration of TNF- $\alpha$ even in pigs fed a diet containing virulent $S$. Typhimurium [19].

Isolation of $S$. Typhimurium $\triangle z n u A B C$ from environmental swabs yielded from pens of vaccinated pigs ended within two weeks. Furthermore, $S$. Typhimurium $\triangle$ znuABC evidenced a shorter viability in pig feces when compared with $S$. Typhimurium ATCC 14028. These findings suggest that $S$. Typhimurium $\triangle z n u A B C$ express a limited persistence in the environment.

The serum concentration of IgG and the antigen specific ex vivo production of IFN- $\gamma$ (supplementary data) by whole blood samples were chosen as parameters to assess the immunogenicity of $S$. Typhimurium $\triangle z n u A B C$. It is known that B-cell and antibodies play a role in the protection from infection in mouse.pigs and humans [20,21]. In particular, antibodies opsonize Salmonella in the early stages of infection, hindering microorganisms to reach their intracellular niche within phagocytes [21,22]. In pigs [23], as well as in humans [24] a humoral immune response is crucial in the early phase of life when an immune response against Salmonella is not developed yet. Nevertheless, in pigs maternal antibodies tend to disappear at the time of weaning, leaving piglets susceptible to Salmonella infection [25]. On that account, S. Typhimurium $\triangle z n u A B C$ proved to prime a humoral immune response inducing high serum levels of IgG.

Live vaccines are believed to be more effective than inactivated ones in controlling S. Typhimurium infection because of their pronounced ability to stimulate the cellular immune compartment and to determine the production of mucosal IgA $[16,25,26]$.

A Th1-oriented immune response is crucial for the complete clearance of Salmonella microorganisms, with activated IFN- $\gamma$ producing $\mathrm{CD}^{+} \mathrm{T}$-cells fostering bacteria killing by monocytes [21]. Although the ex vivo antigen specific release of IFN- $\gamma$ by the whole blood samples of animals vaccinated with $S$. Typhimurium $\triangle z n u A B C$ or inactivated Salmonella vaccine suggests a similar Th1 antigen specific immune response (Fig. S3), we have evidence that after an oral virulent challenge with $S$. Typhimurium ATCC14028, groups vaccinated with $S$. Typhimurium $\triangle z n u A B C$ displayed a greater and more prompt IFN- $\gamma$ production if compared to animals vaccinated with inactivated Salmonella vaccine (data in publication). Oral vaccination of pigs with $S$. Typhimurium $\triangle z n u A B C$ induced serum IgG and evoked a cellular immune response. These data suggest that $S$. Typhimurium lacking the ZnuABC transporter is a promising strain to be used as live vaccine for mucosal delivery in pigs. Furthermore, live vaccines can be administered through drinking water, offering a welfare safeguarding method of vaccination avoiding stressful handling and adverse reactions frequently observed when parenterally injected [27].

Although we proved $S$. Typhimurium $\triangle z n u A B C$ to be immunogenic, vaccinated pigs showed an increase of body temperature and a heterogeneous weight gain that may raise some concerns about its safety. Therefore, the effects in pigs of a reduction of the vaccine dose or the use as vaccines of $S$. Typhimurium strains deleted of ZnuABC and of ZinT [10] and ZupT [28], two other zinc uptake systems, should be investigated. Overall, our data support the safety and immunogenicity of oral administration of $S$. Typhimurium Znu$A B C$ in pigs.

\section{Acknowledgements}

This work was supported by Istituto Superiore di Sanità Intramural Research Project 11 US 24, by European Union Eranet-EMIDA project T99 and by Pfizer Ltd. MR is supported by Public Health Service Grant AI083663.

We acknowledge the great effort of Claudio Barbeno, Mario Pasini, Carlo Giovannelli and Franco Sarra, of the animal facilities; Suzanne Klaus and Janet Z. Liu for the English Language editing of the manuscript. The authors would like to thank the personnel involved in laboratory activities.

\section{Appendix A. Supplementary data}

Supplementary data associated with this article can be found, in the online version, at http://dx.doi.org/10.1016/ j.vaccine.2013.04.032.

\section{References}

[1] Majowicz SE, Musto J, Scallan E, Angulo FJ, Kirk M, O’Brien SJ, et al. International Collaboration on Enteric Disease 'Burden of Illness' Studies 2010;50:882-9.

[2] Foley SL, Lynne AM. Food animal-associated Salmonella challenges: pathogenicity and antimicrobial resistance. J Anim Sci 2008;86:173-87.

[3] Whichard JM, Gay K, Stevenson JE, Joyce KJ, Cooper KL, Omondi M, et al. Human Salmonella and concurrent decreased susceptibility to quinolones and extended-spectrum cephalosporins. Emerg Infect Dis 2007;13:1681-8.

[4] European Food Safety Authority (EFSA) Pires SM, de Knegt L, Hald T. Estimation of the relative contribution of different food and animal sources to human Salmonella infections in the European Union; 2011 http://www.efsa.europa.eu/en/supporting/doc/184e.pdf

[5] Eddicks M, Palzer A, Hörmansdorfer S, Ritzmann M, Heinritzi K. Examination of the compatibility of a Salmonella Typhimurium-live vaccine Salmoporc for three day old suckling piglets. Dtsch Tierarztl Wochenschr 2009;116(7):249-55.

[6] Selke M, Meens J, Springer S, Frank R, Gerlach GF. Immunization of pigs to prevent disease in humans: construction and protective efficacy of a Salmonella enterica serovar Typhimurium live negative-marker vaccine. Infect Immun 2007;75:2476-83.

[7] Xu XG, Zhao HN, Zhang Q, Ding L, Li ZC, Li W, et al. Oral vaccination with attenuated Salmonella enterica serovar Typhimurium expressing Cap protein of PCV2 and its immunogenicity in mouse and swine models. Vet Microbiol 2012;157:294-303.

[8] Leyman B, Boyen F, Van Parys A, Verbrugghe E, Haesebrouck F, Pasmans F. Salmonella Typhimurium LPS mutations for use in vaccines allowing differentiation of infected and vaccinated pigs. Vaccine 2011;29:3679-85.

[9] Ammendola S, Pasquali P, Pistoia C, Petrucci P, Petrarca P, Rotilio G. BattistoniA.High-affinity $\mathrm{Zn} 2+$ uptake system $\mathrm{ZnuABC}$ is required for bacterial zinc homeostasis in intracellular environments and contributes to the virulence of Salmonella enterica. Infect Immun 2007;75:5867-76.

[10] Petrarca P, Ammendola S, Pasquali P, Battistoni A. The Zur-regulated ZinT protein is an auxiliary component of the high-affinity ZnuABC zinc transporter that facilitates metal recruitment during severe zinc shortage. J Bacteriol 2010;192:1553-64.

[11] Liu JZ, Jellbauer S, Poe AJ, Ton V, Pesciaroli M, Kehl-Fie TE, et al. Zinc sequestration by the neutrophil protein calprotectin enhances Salmonella growth in the inflamed gut. Cell Host Microbe 2012;11:227-39.

[12] Pasquali P, Ammendola S, Pistoia C, Petrucci P, Tarantino M, Valente C, et al. Attenuated Salmonella enterica serovar Typhimurium lacking the ZnuABC transporter confers immune-based protection against challenge infections in mice. Vaccine 2008;26:3421-6.

[13] Pesciaroli M, Aloisio F, Ammendola S, Pistoia C, Petrucci P, Tarantino M, et al. An attenuated Salmonella enterica serovar Typhimurium strain lacking the Znu$\mathrm{ABC}$ transporter induces protection in a mouse intestinal model of Salmonella infection. Vaccine 2011;29:1783-90.

[14] Pavic A, Groves PJ, Bailey G, Cox JM. A validated miniaturized MPN method, based on ISO 6579:2002, for the enumeration of Salmonella from poultry matrices. J Appl Microbiol 2010;109:25-34.

[15] Ruckebusch Y, Phaneuf LP, Dunlop R, editors. Physiology of small and large animals. Philadelphia: BC Decker; 1991. p. 388.

[16] Haesebrouck F, Pasmans F, Chiers K, Maes D, Ducatelle R, Decostere A. Efficacy of vaccines against bacterial diseases in swine: what can we expect. Vet Microbiol 2004;100:255-68.

[17] Hotes S, Traulsen I, Krieter J. Salmonella control measures with special focus on vaccination and logistic slaughter procedures. Transbound Emerg Dis 2011;58:434-44.

[18] Husa JA, Edler RA, Walter DH, Holck JT, Saltzman RJ. A comparison of the safety, cross-protection, and serologic response associated with two commercial oral Salmonella vaccines in swine. J Swine Health Prod 2009;17:10-21.

[19] Fraser JN, Davis BL, Skjolaas KA, Burkey TE, Dritz SS, Johnson BJ, et al. Effects of feeding Salmonella enterica serovar Typhimurium or serovar Choleraesuis on growth performance and circulating insulin-like growth factor-I, tumor necrosis factor-alpha, and interleukin-1beta in weaned pigs. J Anim Sci 2007;85:1161-7. 
[20] Griffin AJ, McSorley SJ. Development of protective immunity to Salmonella a mucosal pathogen with a systemic agenda. Mucosal Immunol 2011;4:371-82.

[21] Dougan G, John V, Palmer S, Mastroeni P. Immunity to salmonellosis. Immunol Rev 2011;240:196-210.

[22] Van Parys A, Boyen F, Verbrugghe E, Leyman B, Bram F, Haesebrouck F, et al. Salmonella Typhimurium induces SPI-1 and SPI-2 regulated and strain dependent downregulation of MHC II expression on porcine alveolar macrophages. Vet Res 2012;43:52

[23] Wales AD, Cook AJ, Davies RH. Producing Salmonella-free pigs: a review focusing on interventions at weaning. Vet Rec 2011;168:267-76.

[24] MacLennan CA, Gondwe EN, Msefula CL, Kingsley RA, Thomson NR, White $\mathrm{SA}$, et al. The neglected role of antibody in protection against bacteremia caused by non typhoidal strains of Salmonella in African children. J Clin Invest 2008;118:1553-62.
[25] Hur J, Lee JH. Efficacy of a novel virulence gene-deleted Salmonella Typhimurium vaccine for protection against Salmonella infections in growing piglets. Vet Immunol Immunopathol 2011;139:250-6.

[26] Hur J, Lee JH. Immunization of pregnant sows with a novel virulence gene deleted live Salmonella vaccine and protection of their suckling piglets against salmonellosis. Vet Microbiol 2010;143:270-6.

[27] Mohler VL, Heithoff DM, Mahan MJ, Hornitzky MA, Thomson PC, House JK. Development of a novel in-water vaccination protocol for DNA adenine methylase deficient Salmonella enterica serovar Typhimurium vaccine in adult sheep. Vaccine 2012;30:1481-91.

[28] Karlinsey JE, Maguire ME, Becker LA, Crouch ML, Fang FC. The phage shock protein PspA facilitates divalent metal transport and is required for virulence of Salmonella enterica sv Typhimurium. Mol Microbiol 2010;78: 669-85. 